\title{
STUDY OF THE STIRRING EFFECT IN A TAM2277-205 ISOTHERMAL TITRATION CALORIMETER
}

\author{
M. Rodríguez de Rivera ${ }^{*}$, F. Socorro and J. S. Matos \\ Departamento Física, Universidad de Las Palmas de Gran Canaria, 35017 Las Palmas de Gran Canaria, Spain
}

In this paper, it has been carried out a study to analyze the effect of the stirring velocity in the experimental determination of the mixture enthalpies of several binary mixtures by using a Titration Calorimetric TAM2277-201/2250 by Thermometric AB. The tested liquid mixtures have been ethanol+water and those containing 1-methyl-2-pyrrolidone and (ethanediol, 1,2-propanediol or 1,2-butanediol). The stirring aim is to keep the homogeneity in the mixture process, but the stirring velocity must not be increased in excess in order not to favour the evaporation during the measurement process. This study reveals that every mixture process shows an optimum stirring velocity.

Keywords: binary liquid mixtures, excess molar enthalpies, isothermal titration calorimeter

\section{Introduction}

The isothermal titration microcalorimeters (ITC) are utilized to study solid-liquid and liquid-liquid processes (enthalpies of solution, enthalpies of dilution, enthalpies of reaction in solution, enthalpies of mixing, etc.). In general, the total energy generated in the process that is being studied is determined with these instruments.

When the process is a mixture of liquids, the experimental method consists in the injection of a liquid on other one which is in the mixture cell. There are two ways to use it: (i) The first one consists in injecting a known amount of the liquid on other amount of the liquid, also known. The mixture process produces a calorimetric output signal that, when it returns to experimental zero (baseline), allows to determine the mixture energy by integrating the experimental output (in volts) and dividing by the calorimeter sensitivity (in $\mathrm{V} \mathrm{W}^{-1}$ ). This process goes on until the cell capacity is fulfilled. This way to use it is classical and very widespread [1]. (ii) A second way of using the ITC consists in 'the continuous injection' of a liquid on other at a very low velocity and, after processing the calorimetric signal, it is determined the power that is developed in the mixture process in terms of the concentration [2-4]. This way of ITC operation is not very used due to the difficulty in the mathematical treatment of the calorimetric signal; however, it provides a more detailed thermodynamical information of the thermal process under study.

On the other hand, the calibration of the calorimeters by conduction is always a problem to solve pre- viously to any experimental determination as it is necessary to establish the validity margin of the experimental determinations. In practice, the calibration of the ITC is carried out with electrical calibrations and chemical calibrations using reference mixtures [5].

The quality of the experimental results depends on the consideration of all those aspects that may affect the previous working hypothesis. In previous works, some aspects that can alter the experimental result have been mentioned and described. For the case of conduction, isothermal, continuous injection and variable mass calorimeters, it is important to point out the following aspects:

- Increase of the main time constant when increasing the liquid mass in the mixture cell [6].

- Variation of the sensitivity due to the increase of the liquid level in the mixture cell. This variation can be significant in some cases $[7,8]$.

- Effect that produces the liquid injection that is initially at the thermostat temperature [9].

- Variation of the baseline due to the modification of the thermal coupling between the mixture zone and the thermostat through the stirrer [10].

- Effect that produces the stirring velocity (analyzed in this paper).

These effects and others that could derive from the inherent characteristics of the calorimeter and from the thermal process that is desired to be studied must be taken into account in the initial calibration.

In this paper, it has been carried out an experimental study of the effect of the stirring velocity on the determination of the mixture enthalpies of several

*_Author for correspondence: mrodriguez@dfis.ulpgc.es 
binary systems, using an ITC. For this reason, the following liquid mixtures have been chosen: ethanol+ water (recommended by some authors for the chemical calibration of microcalorimeters) and the binary mixtures of three glycols (ethanediol, 1,2-propanediol and 1,2-butanediol) with 1-methyl 2-pyrrolidone, as the latter ones present a great difference in the dynamic viscosity of their components.

\section{Experimental}

The calorimeter utilized in this study has been an Isothermal Titration Calorimetric TAM2277-201/2250 by Thermometric $\mathrm{AB}$ making use of the stirrer of this experimental device and in combination with the mixture cells of $5 \mathrm{~cm}^{3}$ of capacity (although the advisable maximum volume of liquid is $2.5 \mathrm{~mL}$ [10]). The calorimetric signal is read directly by a digital multimeter HP3457A $( \pm 10 \mathrm{nV})$. The calibration of the calorimeter has already been described in a previous paper [4] and the obtained sensitivity was $391 \pm 9 \mathrm{mV} \mathrm{W}^{-1}$. For the mentioned calibration, the following liquids systems were used: water+ethanol and cyclohexane+ benzene.

To analyze the effect of the stirring velocity in the system ethanol+water, the selected stirring velocities ranged from 0 to $180 \mathrm{rpm}$. At the same time, as it has been previously indicated, it has also been analyzed the stirring effect for the three binary systems 1-methyl-2-pyrrolidone+(ethanediol, 1,2-propanediol, 1,2-butanediol). All measures carried out in this work have been made at $T=298.15 \mathrm{~K}$.

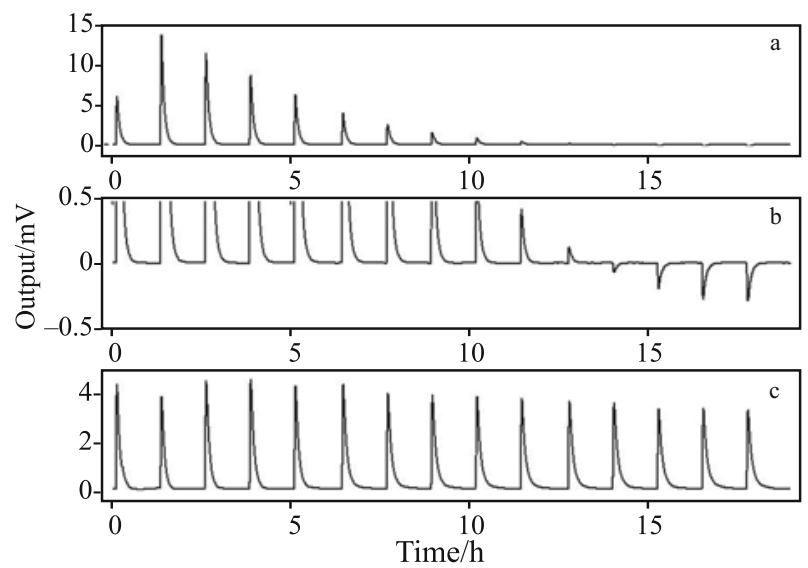

Fig. 1 a - Signal of the experimental output corresponding to 15 injection pulses of ethanol on $1 \mathrm{~cm}^{3}$ of water $(60 \mathrm{rpm}) . \mathrm{b}-$ Extension of the curve a. $\mathrm{c}-$ Signal of the experimental output corresponding to 15 injection pulses of water on $1 \mathrm{~cm}^{3}$ of ethanol (30 rpm). The injection pulses have a duration of $100 \mathrm{~s}$ and the flow is $1 \mu \mathrm{L} \mathrm{s}^{-1}$. In the represented curves, the baseline has been corrected

The experimental method consisted in injecting a total amount of $1.5 \mathrm{~cm}^{3}$ of the first liquid, distributed in injection pulses, on an initial volume of $1 \mathrm{~cm}^{3}$ of the second liquid. The injection flow used in all measures was $1 \mu \mathrm{L} \mathrm{s}^{-1}(\Delta t=1.0 \mathrm{~s})$. Figure 1 shows the experimental output signal corresponding to the cases of injecting ethanol on water and water on ethanol. The baseline noise with the activated stirring was smaller than $\pm 1 \mu \mathrm{V}$.

The injection of the liquid generates an additional calorific power due to the difference between the temperature of the mixture $(T)$ and the temperature of the injected liquid (temperature of the thermostatic bath, $T_{0}$ ). This power responds to the expression $\rho \mathrm{c}_{p} f\left(T-T_{0}\right)$, where $\rho \mathrm{c}_{p}$ is the volumetric heat capacity and $f$ is the injection flow. This effect is very small due to the low injection velocity $\left(1 \mu \mathrm{L} \mathrm{s}^{-1}\right)$, however, this effect has been corrected in all cases. Being $y(t)$ the experimental curve, the corrected curve $y_{\text {corrected }}(t)$ was calculated by the expression [9]:

$$
y_{\text {corrected }}(t)=\left(1+0.0073 \rho c_{\mathrm{p}} f\right) y(t)
$$

where $\left(\rho c_{\mathrm{p}} f\right.$ is in $\left.\mathrm{mW} \mathrm{K}^{-1}\right)$.

For each case, the expression has the following form:

Injecting water:

Injecting ethanol:

Injecting 1-methyl 2-pyrrolidone:

Injecting ethanediol:

Injecting 1,2-propanediol:

Injecting 1,2-butanediol:

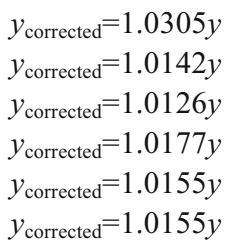

These correcting factors have been determined by relating the liquid heat capacity with the main time constant obtained from the electrical calibration carried out before the injection pulses, i.e., when the cell contains $1 \mathrm{~cm}^{3}$ of pure liquid.

The excess molar enthalpy, $H^{\mathrm{E}}$, is calculated for each molar fraction, $x$, with the following expression [2]:

$$
H^{\mathrm{E}}(x)=\frac{1}{K n_{\mathrm{T}}} \int_{\mathrm{t} 1}^{\mathrm{t} 2} y_{\text {corrected }}(t) \mathrm{d} t
$$

where $K$ is the sensitivity $\left(=391 \mathrm{mV} \mathrm{W}^{-1}\right)$ and $n_{\mathrm{T}}$ is the total number of moles present in the mixture after each injection pulse. The integral is extended between the limits of the injection pulses, taking care that, in the mentioned limits, the experimental output will have to be in the experimental zero. The baseline is corrected in each injection section before doing the integral, since the level of the experimental baseline depends on the stirring velocity and the viscosity of the resulting mixture. 


\section{Results and discussion}

Figure 2 shows the experimental excess enthalpies obtained with different stirring velocities for the binary system ethanol (1)+water (2). In order to make a comparative study, two fixed molar fractions have been chosen: $x_{1}=0.15$ and $x_{1}=0.30$, and the excess enthalpy has been represented for those molar fractions in terms of the stirring velocity (Fig. 3).

When it is injected ethanol on water (Fig. 3a), it is observed that the velocities of 0 and $30 \mathrm{rpm}$ are not sufficient to obtain a homogeneous mixture; however, from $60 \mathrm{rpm}$ the mixture is already homogeneous and the thermodynamical result is the one with the largest absolute value; the increase of the stirring velocity makes the absolute value of the enthalpy begin to decrease due to the possible evaporation. In the case of injecting water on ethanol (Fig. 3b), a stirring velocity of $30 \mathrm{rpm}$ is enough; the increase of the stirring produces liquid evaporation and, as a consequence, a reduction of the absolute value of the mixture enthalpy.

From the previous observations, it can be concluded that, when it is injected ethanol on water, the most adequate stirring velocity is $60 \mathrm{rpm}$; on the other hand, when it is injected water on ethanol, the optimum stirring velocity is $30 \mathrm{rpm}$ up to the molar fraction $x_{1} \approx 0.3$, from which it would have to be increased to $60 \mathrm{rpm}$.

Although the stirring aim is to keep the homogeneity of the liquid mixture, this must not be increased in excess in order not to favour the evaporation during the measurement. However, when ethanediol or propanediol is injected on 1-methyl-2-pyrrolidone and the stirring speed is increased from 120 to $180 \mathrm{rpm}$, we have observed a decrease in the generated total energy of the order of $2 \%$. In addition, the non-convergence of the $H^{\mathrm{E}}$ values, when the compo-

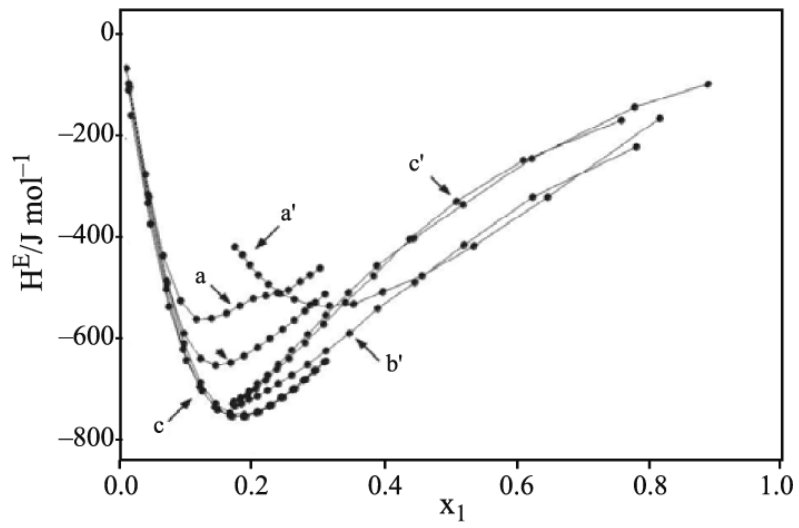

Fig. 2 Excess molar enthalpies of the system ethanol (1)+ water (2) obtained with different stirring velocities; $\mathrm{a}$ - injecting ethanol on water for $0 \mathrm{rpm}, \mathrm{b}$ - for $30 \mathrm{rpm}$, curves $\mathrm{c}$ - for 60,120 and $180 \mathrm{rpm}$; a' - injecting water on ethanol for $0 \mathrm{rpm}$, b' - for $30 \mathrm{rpm}$, curves c' - for 60 and $120 \mathrm{rpm}$ nent 1 or component 2 is injected, is closely related to the different evaporation rates of both components. In a future study, we will try to find correction factors that correct this effect.

The study carried out shows that each mixing process has an optimum stirring velocity. As an exam-
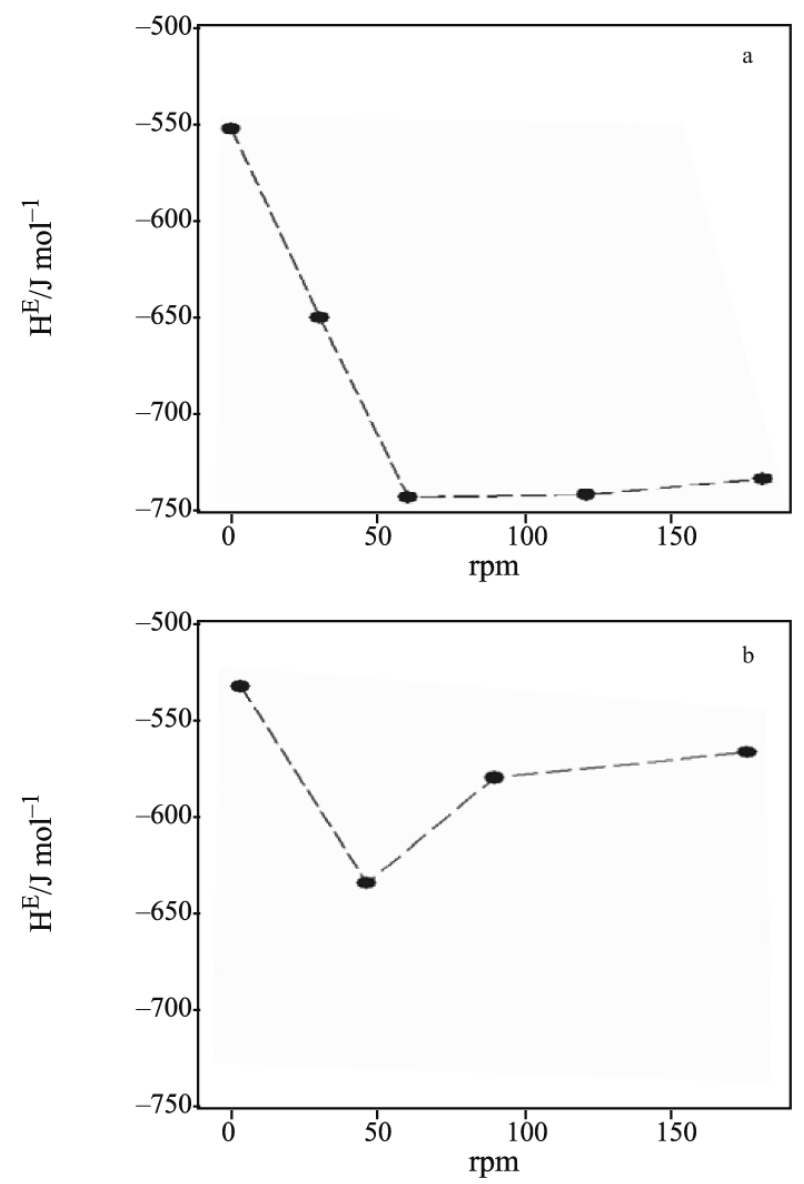

Fig. 3 Excess molar enthalpies obtained with different stirring velocities a - case of injecting ethanol (1) on water (2) for $x_{1}=0.15 ; \mathrm{b}$ - case of injecting water on ethanol for $x_{1}=0.3$

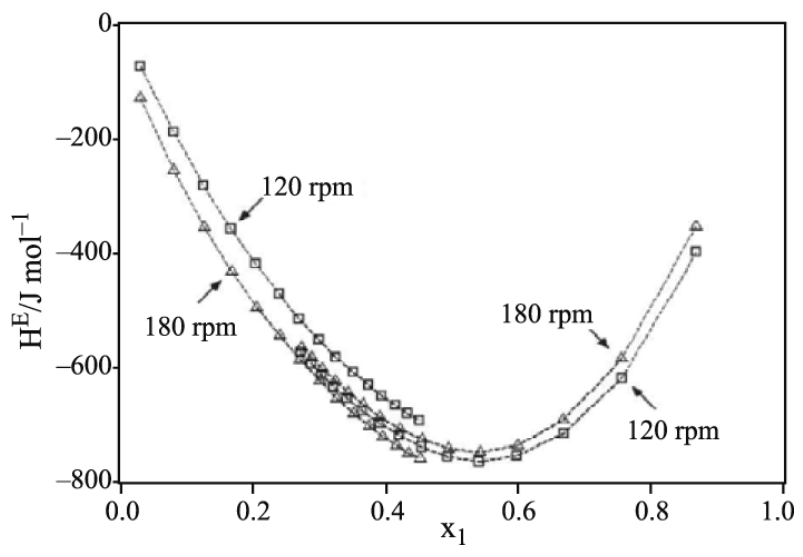

Fig. 4 Excess molar enthalpies for the binary system 1-methyl-2-pyrrolidone (1)+ethanediol (2) using two stirring velocities: $\square-120$ and $\Delta-180 \mathrm{rpm}$ 
Table 1 Coefficients of the Eq. (3) for the binary systems: a - 1-methyl-2-pyrrolidone (1)+ethanediol (2),

b - 1-methyl-2-pyrrolidone (1)+1,2-propanediol (2), c-1-methyl-2-pyrrolidone (1)+1,2-butanediol (2). $\sigma_{\mathrm{d}}$ and $\sigma_{\mathrm{m}}$ are the standard deviation and the maximum deviation, respectively

\begin{tabular}{lcccccc}
\hline Binary system & $A_{0}$ & $A_{1}$ & $A_{2}$ & $A_{3}$ & $\sigma_{\mathrm{d}} / \mathrm{J} \mathrm{mol}^{-1}$ & $\sigma_{\mathrm{m}} / \mathrm{J} \mathrm{mol}^{-1}$ \\
\hline a & -3039.2 & -563.9 & -293.0 & +355.5 & 8.3 & 13.9 \\
b & -1759.4 & -1063.8 & -330.7 & -196.2 & 6.3 & 23.0 \\
c & -1064.7 & -1522.6 & -93.8 & +279.4 & 11.5 & 17.8 \\
\hline
\end{tabular}

ple, and, although the optimum velocity depends on the composition, it can be indicated as stirring velocities acceptable for the system 1-methyl-2-pyrrolidone+ethanediol the following ones: $180 \mathrm{rpm}$ when it is injected 1-methyl-2-pyrrolidone and $120 \mathrm{rpm}$ when the injected liquid is ethanediol (Fig. 4). For the system 1-methyl-2-pyrrolidone+1,2-propanediol: $180 \mathrm{rpm}$ when it is injected 1-methyl-2-pyrrolidone and $120 \mathrm{rpm}$ when it is injected 1,2-propanediol. And, finally, for the system 1-methyl-2-pyrrolidone+ 1,2-butanediol: $60 \mathrm{rpm}$ when it is injected 1-methyl2-pyrrolidone and $180 \mathrm{rpm}$ when it is injected 1,2-butanediol.

With the purpose of extending the observed behaviour, for the whole composition range, of the three binary systems mentioned, we have correlated the observed $H^{\mathrm{E}}$ values with the mole fraction. The correlation was carried out using the unweighed leastsquares method in combination with several equations. However, we report the regression results obtained using the well-known equation by Redlich and Kister:

$$
H^{\mathrm{E}}=x_{1} x_{2} \sum_{\mathrm{i}=0}^{\mathrm{n}} A_{\mathrm{i}}\left(x_{1}-x_{2}\right)^{\mathrm{i}}
$$

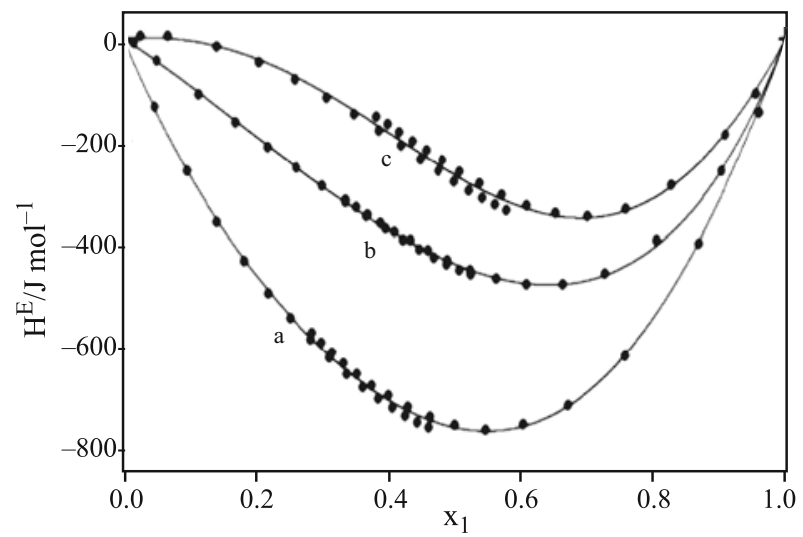

Fig. 5 Excess molar enthalpies $H^{\mathrm{E}}$ as a function of the mole fraction of 1-methyl-2-pyrrolidone for the binary systems: a - 1-methyl-2-pyrrolidone+ethanediol; $\mathrm{b}$-1-methyl-2-pyrrolidone $+1,2$ propanediol; c-1-methyl-2-pyrrolidone $+1,2$ butanediol. - - Experimental results. The continuous lines were obtained from Eq. (3) and the parameters included in Table 1
Table 1 shows the values of the $A_{\mathrm{i}}$ coefficients as well as the statistical parameters derived from the estimate. Figure 5 shows the excess molar enthalpies for each one of the systems at the selected stirring speed.

The other tested equations, even more flexible, did not introduce significant improvements, such as a smaller number of parameters or a smaller standard deviation.

Finally, it is important to point out that, as all liquid mixtures analyzed in this paper are exothermal, the optimum stirring velocity for this type of mixtures will be the one for which the biggest absolute value of the mixture enthalpy will be obtained; i.e. when the mixture process takes place in the whole liquid domain and when the energy due to the evaporation is minimum.

\section{References}

1 A. Ababou and J. E. Ladbury, J. Molecular Recognition, 19 (2006) 79.

$2 \mathrm{H}$. Tachoire and V. Torra, Thermochim. Acta, 110 (1987) 171.

3 F. Socorro, M. Rodríguez de Rivera, J. P. Dubès, H. Tachoire and V. Torra, Meas. Sci. Technol., 1 (1990) 1285.

4 M. Rodríguez de Rivera and F. Socorro, J. Therm. Anal. Cal., 88 (2007) 745.

5 R. Sabbah, An Xu-wu, J. S. Chickos, M. L. Planas Leitão, M. V. Roux and L. A. Torres, Thermochim. Acta, 331 (1999) 93.

6 C. Rey, J. R. Rodríguez, V. Pérez Villar, J. Ortín, V. Torra, J. P. Dubes, R. Kechavarz and H. Tachoire, Thermochim. Acta, 81 (1984) 97.

7 M. Rodríguez de Rivera, H. Tachoire and V. Torra, J. Thermal Anal., 41 (1994) 1392.

8 F. Socorro and M. Rodríguez de Rivera, J. Thermal Anal., 52 (1998) 729.

9 M. Rodríguez de Rivera and F. Socorro, J. Therm. Anal. Cal., 85 (2006) 477.

10 M. Rodríguez de Rivera and F. Socorro, J. Therm. Anal. Cal., 80 (2005) 769.

DOI: $10.1007 / \mathrm{s} 10973-007-8739-7$ 